\title{
Outbreak of Shigella sonnei infections in the Orthodox Jewish community of Antwerp, Belgium, April to August 2008
}

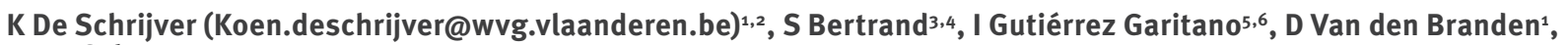

J Van Schaeren ${ }^{7}$

1. Department of Infectious Disease Control, Antwerp, Belgium

2. Department of Epidemiology and Social Medicine, University of Antwerp, Belgium

3. National Reference Centre for Salmonella and Shigella, Scientific Institute of Public Health, Brussels, Belgium

4. Operational Direction for Transmissible and Infectious Diseases, Scientific Institute of Public Health, Brussels, Belgium

5. European Programme for Intervention Epidemiology Training (EPIET), European Centre for Disease Prevention and Control, Stockholm, Sweden

6. Operational Direction for Surveillance and Public Health, Scientific Institute of Public Health, Brussels, Belgium

7. Laboratory of Microbiology Sint Vincentius, Antwerp, Belgium

Citation style for this article:

De Schrijver K, Bertrand S, Gutiérrez Garitano I, Van den Branden D, Van Schaeren J. Outbreak of Shigella sonnei infections in the Orthodox Jewish

community of Antwerp, Belgium, April to August 2008. Euro Surveill. 2011;16(14):pii=19838. Available online: http://www.eurosurveillance.org/ViewArticle.

aspx?Articleld=19838

Article published on 7 April 2011

In the beginning of April 2008 three cases of Shigella sonnei infection were identified among the Orthodox Jewish community of Antwerp, Belgium. We conducted a descriptive study and a household cohort study to identify potential risk factors. Stool samples were cultured and antibiotic susceptibility of the isolates was determined. Between April and August 2008, 42 cases were registered. All characterised isolates $(n=20)$ shared an identical pulsed-field gel electrophoresis profile and were indistinguishable from one of the twelve main strains detected in Israel in 2008, where the index case's father had stayed before the outbreak. The secondary attack rate in households was $8.5 \%$ (95\% confidence interval (Cl): 4.3-12.7). Multivariate analysis identified the following risk factors for secondary spread: households with more than three children (adjusted relative risk (RR): 9.17; $95 \% \mathrm{Cl}: 1.21-69.13)$, children younger than five years (adjusted RR: 5.45; 95\% Cl: 2.44-12.62), and children younger than 12 years assisting in washing younger siblings (adjusted RR: $5.45 ; 95 \% \mathrm{Cl}$ : 2.44-12.17). Rigorous hand washing, use of disposable towels, information for parents and caregivers, and exclusion of symptomatic children from day care, preschool and school for a minimum of 48 hours were implemented.

\section{Introduction}

Infection with Shigella sonnei is a major cause of bacterial gastroenteritis and a leading cause of bacillary dysentery in Belgium [1,2]. Shigellosis is a highly communicable disease and requires a low dose for infection $[1,2]$. In industrialised countries person-to-person transmission accounts for most cases of $S$. sonnei infections, which occur commonly in children aged between six months and 10 years $[2,3]$.
Shigellosis has been a statutorily notifiable disease for clinicians and microbiologists in Belgium since 1971 [4]. Between 2000 and 2009, the number of laboratory isolates of shigellae registered annually by the Belgian reference laboratory of salmonellae and shigellae varied from 316 to 500 . S. sonnei has been the predominant agent causing $65 \%$ to $75 \%$ of all registered Shigella infections $[4,5]$.

In the beginning of April 2008, a microbiologist of one of the town hospitals informed the Department of Communicable Disease Control of Antwerp that S. sonnei had been isolated from three children. The patients belonged to the Orthodox Jewish community of the town. The standardised post-notification interview with their general practitioners and parents showed that the patients had not been out of the country in the month before onset of symptoms. The father of the first case had just returned from a stay in Israel where he felt sick during three days before his return.

Antwerp has an Orthodox Jewish, highly insular community of approximately 10,000 persons living in one quarter of town. The community is characterised by relative social isolation and frequent international contacts especially with New York, London, and Israel [6]. Although sporadic cases of shigellosis have been identified among members of the Orthodox Jewish community in Antwerp before, a well documented outbreak in Belgium has never been described [4].

The first aim of the study was to describe the extent of the outbreak and to identify risk factors for secondary transmission. In addition, we tried to compare the strains from identified cases to confirm that they were genetically indistinguishable and to compare them to the circulating strains in Israel. Using the information 
obtained from these objectives, we wanted to implement appropriate public health control and prevention measures in order to stop the propagation of the disease. An outbreak control team was established to oversee the coordination of this study.

\section{Methods}

\section{Case definition}

A confirmed case was defined as a person living in the town of Antwerp, who had a positive stool culture for S. sonnei in the period between 1 April and 31 August 2008. A probable case was defined as a person who had diarrhoea (three or more loose stools within $24 \mathrm{~h}$ ), fever $\left(\geq 38^{\circ} \mathrm{C}\right)$ and nausea, and who lived in a household where a confirmed case had been detected. An index case in a household or school was the first laboratoryconfirmed shigellosis case in each household or school class. A secondary case was a confirmed or a probable case occurring within seven days after the detection of an index case in a household or in a classroom.

\section{Case finding}

Cases of shigellosis were reported by peripheral microbiological laboratories and clinicians in accordance to statutory notification of infectious diseases. Active case finding among members of affected households and school classes was performed by the local health authorities. General practitioners and paediatricians were asked to report cases to the outbreak control team.

\section{Data collection}

For each identified case, information on demographics, and clinical and microbiological characteristics was collected using a standardised questionnaire. The questionnaire also collected information on possible exposures including any recent travel, attending family gatherings, contacts with other cases, names of household contacts, and attendance at schools or day care centres. It was administered by telephone or by face-to-face interviews at home. Household contacts were followed prospectively for clinical symptoms during one week after contact with the index case. Social Service of the Antwerp Jewish community assisted in contacting people in order to avoid language and cultural barriers. Demographic data collected on household members were compared to data collected from the municipal registry office.

\section{Secondary attack rate study}

To identify specific risk factors for secondary transmission, a retrospective cohort study was conducted among the household contacts of the index cases. A household contact was defined as a person living in the same house as the household index patient. A secondary attack rate was calculated by identifying secondary cases in proportion to the number of household contacts after exclusion of the index case. Potential risk factors for transmission in the household were assessed as follows: the number of children in the household, the age of the children, the presence of children with nappies, the practice of hand washing after washing children, the number of toilets, whether children younger than 12 years (primary school children) were assisting their parents in washing siblings with gastrointestinal symptoms or assisting them at going to the toilet, whether the index case received antimicrobial treatment or whether the index case was admitted to hospital.

\section{Laboratory investigations}

Shigella strains isolated from patients in peripheral clinical laboratories were sent on a voluntary basis to the National Reference Centre for Salmonella and Shigella for serotyping by slide agglutination with commercial antisera (Denka Seiken Co). To evaluate antimicrobial susceptibility, $S$. sonnei specimens were tested by disk diffusion (Kirby-Bauer) following recommendations of the National Committee for Clinical and Laboratory Standards Institute (CLSI), formerly the National Committee for Clinical Laboratory Standards (NCCLS) [7]. Antibiotics tested (BioRad disks) were: ampicillin, amoxicillin/clavulanic acid, cefotaxime, chloramphenicol, tetracycline, naladixic acid, ciprofloxacin, streptomycin, kanamycin, gentamicin, sulfonamides, trimethoprim, and trimethoprim/sulfamethoxazole. Interpretation of inhibition zones was performed according to the CLSI criteria, and quality control was performed using the Escherichia coli ATCC 25922 reference strain [7].

S. sonnei strains were analysed by pulsed-field gel electrophoresis (PFGE) according to the PulseNet method and digested with the restriction endonuclease Xbal (New England Biolabs) [8]. Salmonella enterica serovar Braenderup $\mathrm{H} 9812$ was used as size marker. Fingerprinting II Informatix software (Bionumerics, BioRad) was used to compare the PFGE profiles. Salmonella enterica serovar Braenderup H9812 was used as size marker. Fingerprintingll Informatix software (Bionumerics, BioRad) was used to compare the PFGE profiles. In addition, we included as internal reference five unrelated Shigella strains from national collections that had been isolated from Belgian patients

\section{FIGURE 1}

Onset of illness among Shigella sonnei cases, by week, Jewish community Antwerp, 17 April-31 August 2008 $(n=42)$

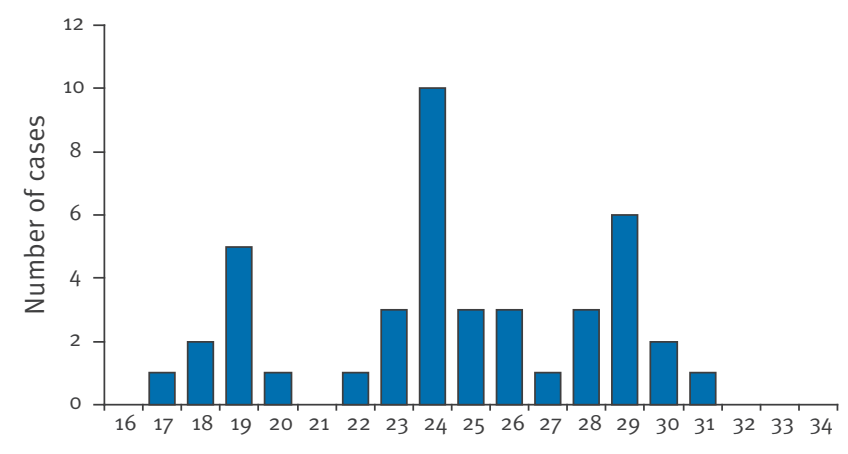

Date (week 2008) 
in 2008. The PFGE profiles of the outbreak strains in Antwerp were also compared to patterns, obtained with the same PulseNet method, of S. sonnei isolated from different orthodox Jewish community outbreaks in Israel between 2000 and 2008. The PFGE gel for them was provided by the Central Laboratories Ministry of Health of Israel. The bands had been analysed using the Dice coefficient and the unweighed-pair group method using average linkage with a tolerance of $1 \%$.

\section{Statistics}

Univariate analysis was performed on data collected in the retrospective household cohort study and crude relative risk (RR) along with $95 \%$ confidence intervals (CI) were calculated to determine associations between potential risk factors and infection. Adjusted RRs were calculated using a binomial regression model. All statistical analyses were performed using Stata software version 11 (StataCorp).

\section{Results \\ Outbreak description}

Between 17 April and 31 August 2008, 42 cases of shigellosis were identified in Antwerp and all of them belonged to the Orthodox Jewish community, with the highest number of cases in week 24 (Figure 1). Thirtytwo of them were confirmed cases and 10 were probable cases. Cases occurred in 19 Jewish families and in four confessional schools. Two additional reported cases of S. sonnei identified outside the Jewish community of Antwerp were excluded from the study because the disease started during a stay in Egypt. They were classified as travel-associated cases.

Of the 42 cases, 20 were male and 22 female. The arithmetic mean of age of cases was 4.4 years with a range from three months to 61 years. Four patients were younger than two years, 19 were between two and five years-old, seven were between six and 10 years-old, six cases were between 11 and 15 years-old, and six cases were older than 20 . The affected families had an average of 4.6 children (range: $1-12$ ). All patients had their residence in an enclosed area in the town centre.

Eighteen cases reported fever $\geq 38^{\circ} \mathrm{C}$ and bloody or mucopurulent diarrhoea and abdominal cramps, and 32 cases were hospitalised. The average duration of illness was eight days with a range from six to 11 days. The average stay at the hospital was 3.4 days.

Of the 42 cases, 15 cases met the criteria for a secondary case. The generation interval was 3.5 days (range from one to four). Three children developed illness within two to five days of detection of a case in a classmate.

\section{Laboratory data}

We received antibiotic susceptibility results from 28 of the 32 confirmed S. sonnei isolates. All of them were resistant to amoxicillin and trimethoprim-sulfamethoxazole, but were susceptible to levofloxacin and

\section{FIGURE 2}

Cluster analysis of PFGE fingerprinting of Shigella sonnei isolated in Antwerp and Israel in 2008

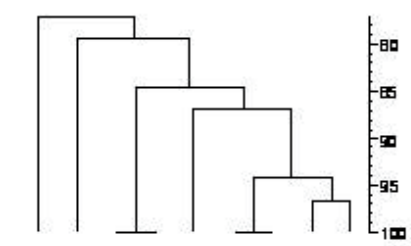

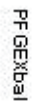
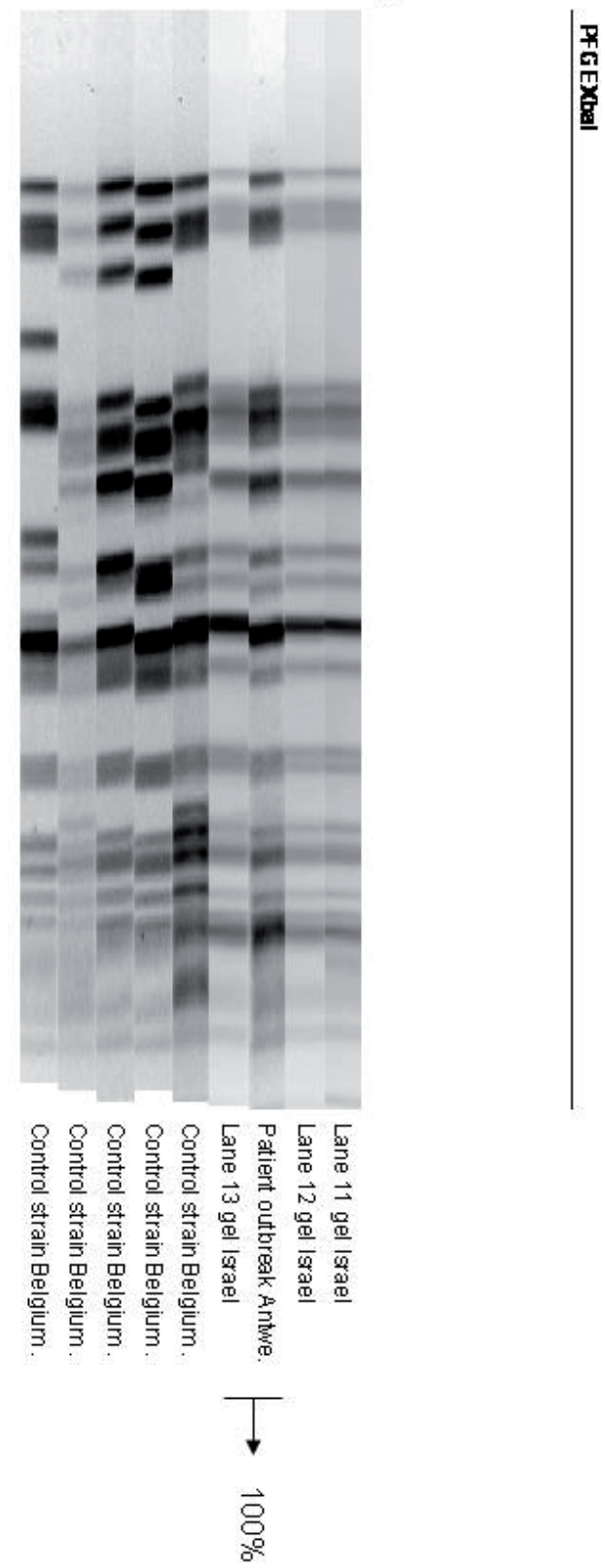

'Lane 11, 12 and 13 gel Israel': S. sonnei from different outbreaks in Orthodox Jewish communities in Israel in 2008.

'Patient outbreak Antwerp': S. sonnei strain from the outbreak in Antwerp in 2008.

'Control strains': unrelated $S$. sonnei strains from the national collection of the Belgian national reference laboratory in 2008 . 
cefotaxim. PFGE was performed on 20 of the 32 isolates and showed that all strains isolated during this outbreak displayed the same restriction-fragment patterns, confirming the relatedness of these isolates. The outbreak strain in Antwerp was compared to 12 different outbreak strains detected in Shigella sonnei shigellosis outbreaks in Orthodox Jewish communities in Israel between 2000 and 2008. Figure 2 presents the results of a cluster analysis on the basis of PFGE fingerprinting of isolates from Antwerp and Israel. The isolate called 'Lane 13 gel Israel', S. sonnei isolated in 2008 in Israel, was indistinguishable from the Belgian outbreak strain. The isolates shown as 'Lane 11 and 12 gel Israel', also isolated in Israel in 2008, had a closely related profile with the Belgian outbreak strain. Five unrelated $S$. sonnei strains originating from national Belgian collections ('Control strain Belgium' from 2008) were used as internal reference.

\section{Secondary attack rate study}

For the 29 affected households with confirmed cases, we identified 175 household contacts, of whom 15 developed shigellosis. A secondary attack rate of 8.5\% (95\% Cl: 4.3-12.7) was calculated. Information on hand washing, the number of toilets in the home and the use of disposable towels was only provided by four of the 25 interviewed households. These questions were excluded in the analysis. The calculated crude and adjusted RRs for the other risk factors are shown in the Table. In the uni- and multivariate analysis, having more than three children in the family, having children younger than 12 years who assisted their parents washing siblings and helping them go to the toilet, and having children younger than five years, were significantly associated with a higher risk of secondary transmission. Having more than three children in the household was associated with the highest risk, with an adjusted RR of 9.17 (95\% Cl: 1.21-69.13). Hospitalisation and treatment with antibiotics of the household index cases were not significantly associated with a lower risk of secondary infection, with a respectively adjusted $\mathrm{RR}$ of 0.88 (95\% $\mathrm{Cl}: 0.61-3.1)$ and an adjusted RR of 1.8 (95\% Cl: $0.80-4 \cdot 34)$.

\section{Control measures}

To prevent further spread of the disease, parents of the affected families were advised of the importance of hand washing with running water and liquid soap after using the toilet or washing the children and also on the importance using disposable towels and cleaning the toilets with chlorine. The need to decontaminate toys was highlighted. In June 2008 educational presentations for parents, caregivers and teachers were organised. Information was also published in the local media. Physicians were informed via articles in the local medical infectious disease journal. Schools were informed on the hygiene of hand washing facilities. We insisted on excluding symptomatic children for a minimum of 48 hours after clinical recovery from day care centres, preschool and school attendance [9].

\section{Discussion}

We identified a cluster of 42 cases of shigellosis in the Orthodox Jewish community of Antwerp with 32 isolates laboratory-confirmed as S. sonnei with the same genetic profile. Temporal and spatial clustering in one area of town affecting one specific community supported the hypothesis of a single ongoing outbreak, maintained through person-to-person transmission. Statutory laboratory-based surveillance of shigellosis failed to identify concurrent cases outside this community. Two additional $S$. sonnei cases notified in the study period in the province of Antwerp in people who were not Jewish were most probably not linked to the outbreak. The disease started during a stay in Egypt and they were classified as travel-associated cases.

The index case was most probably infected by their father, who had suffered from gastrointestinal problems during a stay in Tel-Aviv, Israel until two days before symptom onset in the index case but did not seek medical care. No exceptional family gatherings could be identified except for synagogue attendance. The father also reported having been in contact with relatives coming from London.

To investigate a possible link between the outbreak in Antwerp and an ongoing outbreak in Israel [10], the circulating strains in both outbreaks were compared. Such a link was supported by the microbiological analysis in which the main strain circulating in Israel at the time and the outbreak strain in Antwerp were indistinguishable. The father of the index case also reported having been in contact with relatives coming from

\section{TABLE}

Risk factors of illness among household contacts of an index case with shigellosis, Jewish community Antwerp, 17 April-31 August $2008(n=42)$

\begin{tabular}{|c|c|c|c|c|}
\hline \multirow[b]{2}{*}{ Exposure } & \multicolumn{2}{|c|}{ Univariate analysis } & \multicolumn{2}{|c|}{ Multivariate analysis } \\
\hline & Crude relative risk & $95 \%$ confidence interval & Adjusted relative risk & $95 \%$ confidence interval \\
\hline >3 children in household & 8.47 & $1.14-62.98$ & \begin{tabular}{|l|l}
9.17 & \\
\end{tabular} & $1.21-69.13$ \\
\hline Children with nappies & 2.41 & $0.90-6.48$ & 1.59 & $0.84-3.01$ \\
\hline Children $<5$ years in household & 6.0 & $1.39-25.80$ & 5.24 & $1.17-23.62$ \\
\hline Children «12 years assisting parents washing siblings & 6.54 & $2.59-16.51$ & 5.45 & $2.44-12.17$ \\
\hline Index case in household hospitalised & 1.02 & $0.38-2.75$ & 0.88 & $0.61-3.10$ \\
\hline Index case in household treated with antibiotics & 1.42 & $0.50-3.99$ & 1.87 & $0.80-4 \cdot 34$ \\
\hline
\end{tabular}


London. Addiman et al. reported on an outbreak of shigellosis in London starting a month before the onset of our outbreak in Antwerp in 2008 [11]. A strain from the outbreak of London 2008 could not be obtained for comparison.

Outbreaks of shigellosis with $S$. sonnei and recurrent increases in the number of cases in Orthodox Jewish populations have already been notified in 2008 and before in different countries. Calderon-Margalit et al. showed that between 1998 and 2006, outbreaks of shigellosis followed a biennial pattern in Israel with annual rates that ranged from 18 to 353 cases per 100,000 population [12]. Also in 2009 outbreaks of $S$. sonnei in Israel were still continuing [10]. Close contacts, day care attendance and having many young children in the families were considered risk factors. The characteristics of the outbreak in Antwerp are comparable with prolonged outbreaks of $S$. sonnei reported by Sobel et al. in North America in traditionally observant Jewish communities between 1994 and 1996 [13] , with outbreaks reported by Garret et al. in New York in 2005 [14] and with the outbreak in London in 2008 [11].

The secondary attack rate of $8.5 \%$ found in this study is comparable to those noticed in other studies $[2,15]$. In larger studies, secondary attack rate differed according to age and to the species of bacterium [2]. Due to the limited number of cases in our study, agespecific attack rates could not be calculated. Dupont et al. showed that for one to four year-olds, the secondary attack rate can reach $40 \%$ [2]. The combination of high communicability due to the low infective dose, crowding, and frequent contacts are known explanations for the high secondary attack rate for shigellosis $[2,14,15]$. In our study we analysed specific risk factors which might explain the noted secondary attack rate. Having more than three children in the household and having children younger than five years of age was significantly associated with the occurrence of secondary cases, which is consistent with data from other authors [14]. Contrary to what we expected, having children with nappies in the household was not a significant independent risk factor in our study. This could be due to good hygienic habits of the adults when providing care for their babies. That the index case of the family was hospitalised was hypothesised to be a protective factor, but the adjusted RR was 0.88 (95\% Cl: $0.61-3.10)$. The low number of cases and the different intervals between onset of the disease and moment of hospitalisation of the cases might have interfered with the association. Being treated with antibiotics was not significantly associated with a lower risk of secondary transmission either. Different delays in the start of therapy, the broad spectrum of used antibiotics and the small number of cases might explain the calculated RR of 1.8 (95\% Cl: $0.80-4 \cdot 34)$.

However, it was remarkable that children younger than 12 years, helping their parents take care of babies, was associated with a higher risk of secondary cases (adjusted RR: 5.45 ; 95\% Cl: 2.44-12.17). In families with a high number of children, older children were asked to help. There is a risk that these young children are less sensitive or less knowledgeable than their parents on the risk and the practice of hand hygiene.

Visiting friends and relatives in areas with higher risk of shigellosis might be the seeding event leading to shigellosis outbreaks in especially susceptible communities. This is the case for Jewish communities in Antwerp that are more susceptible due to the high number of children in the families, the many social contacts, living in a relatively small community, and the frequent contact with relatives who live in areas of higher endemic prevalence of shigellosis, like certain neighbourhoods in London or Israel $[11,12]$.

The high hospital admission rate in our study (32 of 42 cases) suggests that we have probably detected only the most severe cases, whereas milder cases could also have been expected. Presenting bloody or mucopurulent diarrhoea was noted in 18 of the cases. This is unusual compared to the expected picture of a S. sonnei infection which is normally associated with a milder disease $[2,3,5]$. We have therefore reason to consider under-diagnosis and under-reporting in this outbreak, which is also mentioned in similar outbreaks of shigellosis elsewhere [2].

Several limitations of the study especially for the secondary attack rate study should be noted. Firstly, the number of cases was limited. This raises concerns about the interpretation of the calculated relative risks. Secondly, we assumed that secondary cases acquired their infection at home. Alternatively they might have been infected during pre-school and school attendance or by visiting friends and relatives. Thirdly, personal questions like 'did you wash your hands after toilet use?' and 'how many toilets do you use at home' were often not answered, most probably due to their sensitive and private nature [16]. It is likely that not all possible risk factors could be explored in the study of this outbreak.

Early notification of shigellosis enabled prompt reaction, and implementation of the advice most probably put a stop to further propagation. We presume that especially the intensive hand washing campaign in families and schools, the educational presentations and specific information to physicians contributed to stopping the outbreak.

PFGE in studies of clusters has been shown to be a highly effective method of characterising S. sonnei and an important tool for outbreak investigations [17]. Provided that the same protocol is used, it allows comparison of strains detected in different outbreak. The genetic relatedness of the strains in this study provides strong evidence that this cluster was a single outbreak and associated with recurrent endemic shigellosis in Israel $[10,12]$. 
In conclusion, this is the first well-documented outbreak of $S$. sonnei in the Orthodox Jewish community of Antwerp and Belgium for which a direct link to an ongoing outbreak of endemic shigellosis in Israel could be identified. The combination of case finding, source tracing, and comparing different strains with PFGE was essential for confirming the hypothesis of import of an outbreak strain from Israel into the local community, and implementation of hand washing was important to stop the propagation of the epidemic.

\section{Acknowledgements}

We thank physicians, microbiologists and public health nurses for their collaboration during the study. We thank Emmanuel Robesyn for his support during the analysis of the data, Annick Lenglet for the critical comments, and we are grateful for the assistance of the Social Service of the Jewish community of Antwerp for contacting people. We also thank Lea Valinsky from the Central Laboratories Ministry of Health, Jerusalem Israel who provided the PFGE pictures of different outbreak strains detected in Israel.

\section{References}

1. Guerrant RL, Steiner TS. Principles and syndromes of enteric infection. In: Mandell GL, Benett JE, Dolin R, eds. Mandell, Douglas, and Benett's Principles and Practice of Infectious Diseases. 6th ed. Philadelphia: Churchill Livingstone; 2005. p. 1215-31.

2. Dupont HL. Shigella species. In: Mandell GL, Benett JE, Dolin R, eds. Mandell, Douglas, and Benett's Principles and Practice of Infectious Diseases. 6th ed. Philadelphia: Churchill Livingstone; 2005. p. 2655-61.

3. Thielman NM, Guerrant RL. Acute infectious diarrhea. N Engl J Med. 2004;350(1):38-47.

4. De Schrijver K, Bertrand S, Van Den Branden D, Van Schaeren J, Van Meervenne E, Van De Staey W, et al. Shigelloseclusters in Antwerpen, Is 'den rooden loop' terug in het land? [Clusters of shigellosis. Is dysentery back in town?] Vlaams Infectieziekte Bulletin 70/2009/4. 2009. [Dutch]. Available from: http://www. infectieziektebulletin.be/defaultSubsite.aspx?id=20188

5. Ducoffre G. Surveillance des Maladies Infectieuses par un Réseau de Laboratoires de Microbiologie 2009. Tendances Epidémiologiques 1983-2008. [Infectious disease surveillance by a network of microbiology laboratories 2009. Epidemioligical trends 1983-2008]. Brussels: Institut Scientifique de Santé Publique; 2009. [French]. Available from: http://www.wiv-isp.be/epidemio/epinl/plabnl/plabannl/ indexo9.htm

6. Albicht L. De Joden in Antwerpen. [The Jews in Antwerp]. Antwerpen: Houtekiet; 2004. [Dutch]

7. Clinical and Laboratory Standards Institute. Performance Standards for Antimicrobial disk Susceptibility tests, Approved Standard. 9th edition. Wayne; CLSI Standards; 2006.

8. PulseNet. Atlanta: Centers for Disease Control and Prevention; Feb 2011. Available from: http://www.cdc.gov/pulsenet

9. Hawker J, Begg N, Blair I, Reintjes R, Weinberg J. Shigella. In: Communicable Disease Control Handbook. Oxford: Blackwell Science; 2001:181-4.

10. Cohen D, Bassal R, Valinski L, Vasilev G, Green MS for the Shigella Surveillance Network. Cyclic occurence of epidemics of Shigella sonnei shigellosis in Israel. Vaccines for Enteric Diseases; 9-11 Sept 2009; Malaga, Spain. Available from: http://www.meetingsmanagement.com/ved_2009/day_2.htm

11. Addiman S, Kitching A, Figueroa J, Saliba V, Nicholas M, Cheasty T, et al. Is history repeating itself? Shigella sonnei PT P outbreak in the Orthodox Jewish community in Hackney, London. Health Protection 2008 conference; 15-17 September 2008; Warwick, United Kingdom. Poster presentation.

12. Calderon-Margalit R, Navon-Venezia S, Gefen D, Amitai Z, Barda R, Vulikh I, et al. Biennal hyperendemic shigellosis in observant Jewish community. Epidemiol Infect. 2010;138(2):244-52.
13. Sobel J, Cameron DN, Ismail J, Strockbine N, Williams M, Diaz PS, et al. A prolongued outbreak of Shigella sonnei in traditionally observant Jewish communities in North America caused by a molecular distinct bacterial subtype. J Infect Dis. 1998;177(5):1405-9.

14. Garret V, Bornschlegel K, Lange D, Reddy V, Kornstein L, Kornblum J, et al. A recurring outbreak of Shigella sonnei among traditionally observant Jewish children in New York city: the risks of daycare and household transmission. Epidemiol Infect. 2006;134(6):1231-6.

15. Jonsson J, del Álvarez-Castillo MC, Sanz JC, Ramiro R, Ballester $\mathrm{E}$, Fernánez $\mathrm{M}$, et al. Late detection of a shigellosis outbreak in a school in Madrid. Euro Surveill. 2005;10(10): pii=571. Available from: http://www.eurosurveillance.org/ViewArticle. aspx?Articleld $=571$

16. Curtis V, Cairncross S. Effect of washing hands with soap on diarrhoea risk in the community: a systematic review. Lancet Infect Dis. 2003;3(5):275-81.

17. Litwin CM, Leonard RB, Carrol KC, Drummond WK, Pavia AT. Characterization of endemic strains of Shigella sonne by use of plasmid DNA analysis and pulsed field gel electrophoresis to detect patterns of transmission. J Infect Dis. 1997;175(4):864-70. 